\title{
Use of webMathematica for optics training over the Web
}

James Wyant

James C. Wyant, "Use of webMathematica for optics training over the Web," Proc. SPIE 9663, Eighth International Topical Meeting on Education and Training in Optics and Photonics, 96630J (6 October 2003); doi: $10.1117 / 12.2208494$

SPIE Event: Eighth International Topical Meeting on Education and Training in Optics and Photonics, 2003, Tucson, Arizona, United States 


\title{
Use of webMathematica for optics training over the Web
}

\author{
James C. Wyant \\ Optical Sciences Center, University of Arizona, Tucson, AZ 85721 \\ telephone: 520-621-2448; e-mail: jcwyant@optics.arizona.edu
}

\begin{abstract}
Mathematica provides a good mathematical and graphical mechanism for using a web browser to study optics problems over the internet. Techniques for using webMathematica to study interference, Fraunhofer and Fresnel diffraction, polarization, and Seidel and Zernike aberration will be described and illustrated. (See http://wyant.optics.arizona.edu/math.htm).

(C)2003 Optical Society of America

OCIS codes: (120.3180) Interferometry; (050.1940) Diffraction
\end{abstract}

\section{Summary}

Mathematica is very powerful for modeling optics problems because of its symbolic nature and the vast number of mathematical functions it contains and its graphics capability. However, because Mathematica has so many capabilities it takes a fair amount of work to become an expert using Mathematica. For this reason an optics user may get so involved in the Mathematica part of the problem that he loses sight of the optics that is being studied. However, most people are experts at using a web browser, so if Mathematica is used in such a way as to hide all the Mathematica complexity behind a web browser and present an optics user with the ability to vary the optical parameters of interest it is possible to study rather complex optical problems without being an expert at using the mathematical program used to solve the optical problem. Furthermore, if the Mathematica software is placed on a web server the computers running the web browser do not need to have Mathematica installed and the optics problem can be solved from any place where an internet connection is available.

webMathematica makes it possible to solve mathematical optics problems over the internet without having any software other than a modern version of the web browser. This presentation will describe the procedure used to set up webMathematica to solve optics problems and examples solving diffraction, interference, polarization and aberration problems will be given. The webMathematica capability for studying optics problems can be seen at http://wyant.optics.arizona.edu/math.htm.

\section{References}

Tom Wickham-Jones, webMathematica, http://www.wolfram.com/products/webmathematica/index.html. 